\title{
A nomogram constructed using intraoperative ex vivo shear-wave elastography precisely predicts metastasis of sentinel lymph nodes in breast cancer
}

\author{
Soong June Bae ${ }^{1}$ Ji Hyun Youk ${ }^{2}$. Chang Ik Yoon ${ }^{3}$. Soeun Park ${ }^{1}$. Chi Hwan Cha ${ }^{1} \cdot$ Hak Woo Lee ${ }^{1}$. Sung Gwe Ahn ${ }^{1}$. \\ Seung Ah Lee ${ }^{4}$ Eun Ju Son ${ }^{2} \cdot$ Joon Jeong ${ }^{1}$ (it)
}

Received: 21 June 2019 /Revised: 28 August 2019 / Accepted: 20 September 2019/Published online: 6 November 2019

(C) The Author(s) 2019

\begin{abstract}
Objective To develop a nomogram and validate its use for the intraoperative evaluation of nodal metastasis using shear-wave elastography (SWE) elasticity values and nodal size

Methods We constructed a nomogram to predict metastasis using ex vivo SWE values and ultrasound features of 228 axillary LNs from fifty-five patients. We validated its use in an independent cohort comprising 80 patients. In the validation cohort, a total of 217 sentinel LNs were included.

Results We developed the nomogram using the nodal size and elasticity values of the development cohort to predict LN metastasis; the area under the curve (AUC) was 0.856 (95\% confidence interval (CI), 0.783-0.929). In the validation cohort, $15(7 \%)$ LNs were metastatic, and $202(93 \%)$ were non-metastatic. The mean stiffness (23.54 and $10.41 \mathrm{kPa}, p=0.005)$ and elasticity ratio (3.24 and 1.49, $p=0.028)$ were significantly higher in the metastatic LNs than those in the non-metastatic LNs. However, the mean size of the metastatic LNs was not significantly larger than that of the non-metastatic LNs $(8.70 \mathrm{~mm}$ vs 7.20 $\mathrm{mm}$, respectively; $p=0.123$ ). The AUC was 0.791 (95\% CI, 0.668-0.915) in the validation cohort, and the calibration plots of the nomogram showed good agreement.

Conclusions We developed a well-validated nomogram to predict LN metastasis. This nomogram, mainly based on ex vivo SWE values, can help evaluate nodal metastasis during surgery.

\section{Key Points}

- A nomogram was developed based on axillary LN size and ex vivo SWE values such as mean stiffness and elasticity ratio to easily predict axillary LN metastasis during breast cancer surgery.

- The constructed nomogram presented high predictive performance of sentinel LN metastasis with an independent cohort.

- This nomogram can reduce unnecessary intraoperative frozen section which increases the surgical time and costs in breast cancer patients.
\end{abstract}

Keywords Breast neoplasm $\cdot$ Elasticity imaging techniques $\cdot$ Nomogram $\cdot$ Lymphatic metastasis

Joon Jeong

gsjjoon@yuhs.ac

1 Department of Surgery, Gangnam Severance Hospital, Yonsei University College of Medicine, 211 Eonju-ro, Gangnam-gu, Seoul 06273, Republic of Korea

2 Department of Radiology, Gangnam Severance Hospital, Yonsei University College of Medicine, Seoul, Republic of Korea

3 Department of Surgery, St Mary's Hospital, College of Medicine, The Catholic University of Korea, Seoul, Republic of Korea

4 Department of Surgery, CHA Bundang Medical Center, CHA University, Seongnam, Republic of Korea

\author{
Abbreviations \\ ALND Axillary lymph node dissection \\ AUC Area under the curve \\ ER Estrogen receptor \\ HER2 Human epidermal growth factor receptor 2 \\ HG Histologic grade \\ LN Lymph node \\ ROI Region of interest \\ SLNB Sentinel lymph node biopsy \\ SWE Shear-wave elastography
}




\section{Introduction}

Axillary lymph node (LN) metastasis is one of the important prognostic factors in breast cancer $[1,2]$. Sentinel LN biopsy (SLNB) has become the standard method for axillary staging in patients with clinically negative nodes [3]. Further, intraoperative pathologic assessment of sentinel LNs enables surgical staging and aids in surgical decision-making regarding whether axillary LN dissection (ALND) should be performed during surgery [4-7]. However, it requires skilled pathologists and equipment, which increases the surgical time and costs.

Shear-wave elastography (SWE) can quantitatively calculate the elasticity parameters of target lesions and provide values, such as mean, minimum, and maximum stiffness; standard deviation of elasticity; and elasticity ratio of breast lesions to the adjacent fat tissue. It is helpful in distinguishing breast cancerous lesions because malignant tissues tend to have increased stiffness compared with benign tissues [8-12]. Also, SWE well predicted the histological upgrade to invasive cancer in ductal carcinoma in situ confirmed at biopsy and response to neoadjuvant chemotherapy in breast cancer $[13,14]$. Furthermore, several studies reported that SWE could be applied to identify axillary LN metastasis as well as breast malignancy [15-19]. In addition, we previously reported that the nodal size and elasticity values, such as maximum stiffness, mean stiffness, and elasticity ratio of axillary LNs to the adjacent fat tissues, were associated with metastatic axillary LNs [20]. Therefore, ex vivo SWE is thought to be a feasible method to predict axillary LN metastasis.

Based on these results, we hypothesized that the nomogram constructed using ultrasound features and elasticity values measured via intraoperative ex vivo SWE can accurately predict nodal metastasis. Furthermore, the disadvantages of intraoperative frozen section analysis may be compensated if the nomogram precisely predicts sentinel LN metastasis, since ex vivo SWE can be performed easily, and no additional equipment is required, except for ultrasound. For this reason, we developed and validated a nomogram to predict the sentinel LN metastasis using ultrasound features and ex vivo SWE values in this study.

\section{Materials and methods}

\section{Patients}

In our previous prospective study, a total of 228 axillary LNs, including sentinel LNs obtained from fifty-five patients who underwent breast cancer surgery in Gangnam Severance Hospital from May 2014 to April 2015, were investigated [20]; these patients were utilized as the development cohort. Another 80 patients diagnosed with breast cancer underwent breast cancer surgery at the same hospital from August 2015 to March 2016 were prospectively enrolled. Patients with stage IV cancer or those who received neoadjuvant chemotherapy were excluded. Unlike in the development cohort, 217 sentinel LNs obtained from these 80 patients examined using intraoperative ex vivo SWE were included in the validation cohort.

Our study was approved by the Institutional Review Board of Gangnam Severance Hospital and conducted in accordance with the Good Clinical Practice guidelines and the Declaration of Helsinki principles.

\section{SLNB}

SLNB was performed using 99mTc-labeled tin colloid. Intradermal injection of $0.4 \mathrm{~mL} 30 \mathrm{MBq}(0.8 \mathrm{mCi}) 99 \mathrm{mTc}$ tin colloid diluted in normal saline solution was performed at four peri-areolar sites. Sentinel LNs were determined by employing a gamma detector (Gamma Detection System, Neoprobe Corporation). The LNs showing a high radioactivity were dissected, after which the gamma detector was used again to confirm the correct sentinel LNs. All radioactive LNs with an account equal to or greater than $10 \%$ of the highest radioactive $\mathrm{LN}$ were removed. When suspicious LNs not detected by the gamma probe were found after SLNB, they were removed and examined as non-sentinel LNs. If the result of frozen pathology was positive for malignancy, ALND was performed.

\section{B-Mode ultrasound with ex vivo SWE}

Excised LNs were delivered to the radiology part. Then, B-mode ultrasound with ex vivo SWE was performed as mentioned in our previous study [20]. In summary, the nodal size and elasticity values, such as mean stiffness and elasticity ratio of the sentinel LNs to the adjacent fat tissues, were examined during surgery via B-mode ultrasound and SWE using the Aixplorer ultrasound system (Super Sonic Imagine) and ShearWave ${ }^{\mathrm{TM}}$ elastography mode $[21,22]$ with a $4-15-\mathrm{MHz}$ linear transducer. During the examination, the radiologists need to handle the specimen carefully so as not to exert pressure on the excised LNs by the ultrasound transducer and sufficient contact jelly was used to avoid artificial stiffness. The longest diameter of the $\mathrm{LN}$ which was hypoechoic compared with the adjacent fat tissue was measured in B-mode ultrasound (Fig. 1). After few seconds of immobilization to allow the SWE image to stabilize, mean stiffness was measured by placing a fixed 2-mm circular region-of-interest (ROI) box (Q-BOX ${ }^{\mathrm{TM}}$; SuperSonic Imagine) on the stiffest region of the excised LNs. The elasticity ratio was calculated automatically when the first ROI was placed on the stiffest region of excised LNs, and the other ROI was immediately placed on the adjacent fat tissue (Fig. 1). Four radiologists with a clinical experience of more than 2 years performed B-mode ultrasound with SWE, 


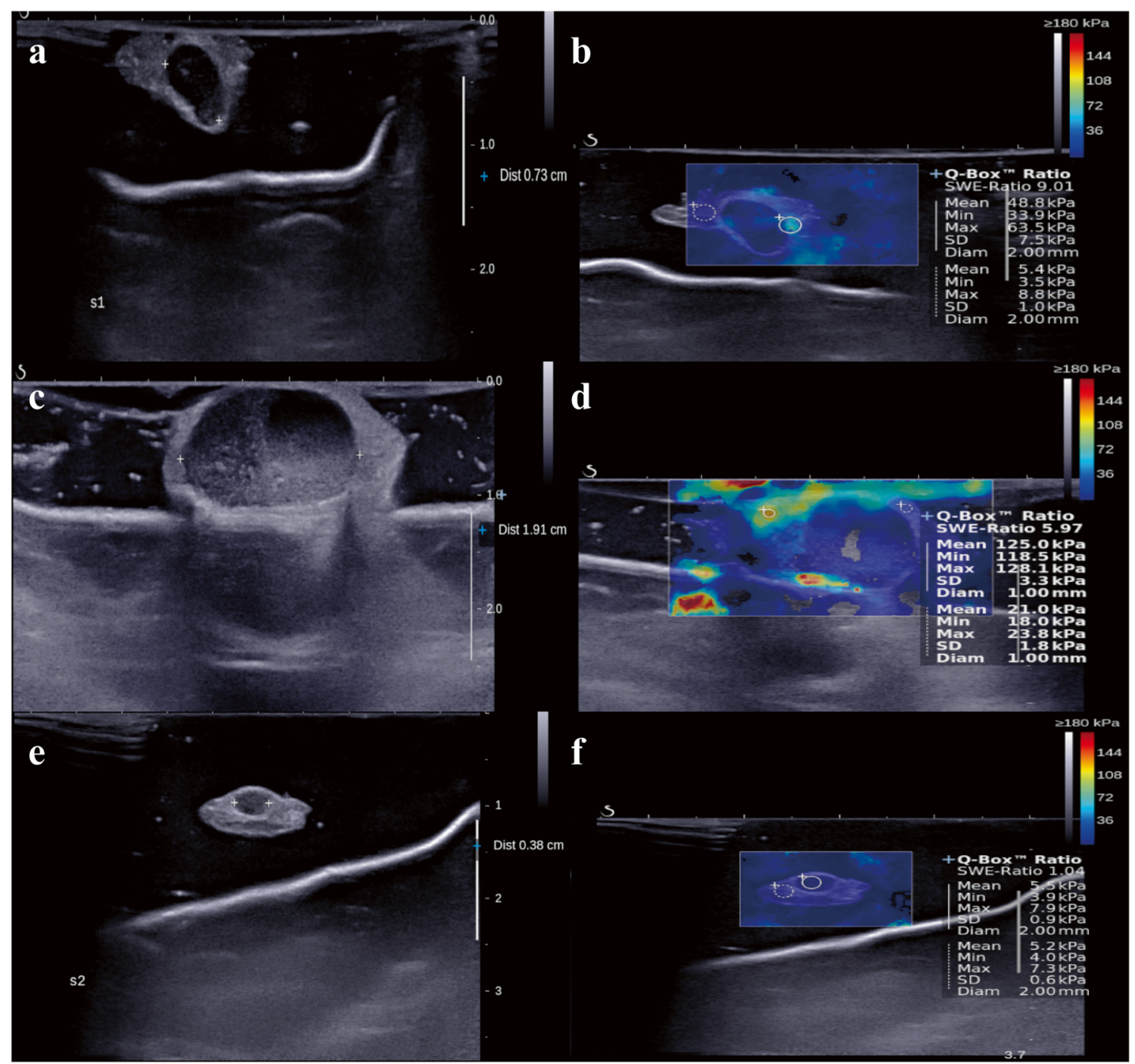

Fig. 1 B-Mode ultrasound and shear-wave elastography (SWE). a BMode ultrasound showed $0.73-\mathrm{cm}-$ sized excised sentinel lymph node with adjacent fat tissue. b The mean stiffness was measured by placing the 2-mm-sized region-of-interest (ROI) on the stiffest part of the excised sentinel lymph node (circle). The stiffness of the adjacent fat tissue was measured by placing another ROI on the surrounding fat tissue (dotted circle). Then, the elasticity ratio was calculated automatically. a-d BMode ultrasound and shear-wave elastography (SWE) images of patients with lymph node metastasis. e, f B-Mode ultrasound and shear-wave elastography (SWE) images of patient without lymph node metastasis and one radiologist with more than 10 years of clinical experience reviewed the results, i.e., nodal size and elasticity values, such as mean stiffness and elasticity ratio.

\section{Pathologic evaluation of excised LNs}

After the radiologic examination was finished, the pathologic evaluation of sentinel LNs was performed. The excised sentinel LNs were cut into 2-3-mm sizes, and all slices were assessed. The size of the metastatic lesions within the excised nodes was reported in accordance with the staging system of the American Joint Committee on Cancer as follows: $>2 \mathrm{~mm}$, macro-metastasis; $0.2-2.0 \mathrm{~mm}$, micro-metastasis; and $<0.2$ $\mathrm{mm}$, isolated tumor cells [23]. In this study, sentinel LN metastasis was defined as macro-metastasis.

\section{Development of the nomogram and statistical analysis}

A nomogram was established on the basis of the results of the multivariate analysis in the development cohort. Thereafter, 200 bootstrap samples were used for internal validation of the development cohort, and external validation was performed with an independent cohort. The performance of the constructed nomograms was quantified with respect to discrimination and calibration [24]. The discriminative power of whether the constructed nomogram can correctly predict 
the probability of LN metastasis was quantified using the area under the receiver operating characteristic curve. Calibration was performed to identify the agreement between the observed outcomes and predicted probabilities of metastasis among the excised LNs. To verify the suitability of the constructed nomograms, the Hosmer-Lemeshow goodness-of-fit test was performed $[25,26]$. The software used to perform these analyses was the SAS program (ver 9.2, SAS Institute Inc.) or the R Statistical Package (ver 3.3.3, Institute for Statistics and Mathematics).

The characteristics of the patients were analyzed using the chi-square test and independent two-sample $t$ test. The mean elasticity values were compared between the non-metastatic and metastatic sentinel LNs using the independent two-sample $t$ test. Analysis was performed using the SPSS software ver 23 (SPSS).

\section{Results}

\section{Nomogram construction using the development cohort and internal validation}

We previously reported the characteristics and elasticity values of the harvested axillary LNs measured using B-mode ultrasound and intraoperative ex vivo SWE in the development cohort [20]. Briefly, the nodal size and elasticity values, such as mean stiffness and elasticity ratio, were associated with axillary LN metastasis in the multivariable analysis. The nomogram was constructed on the basis of significant factors from the multivariable analysis in the development cohort to predict the probability of axillary LN metastasis (Fig. 2a). From binary logistic regression model for multivariable analysis, we obtained intercept and regression coefficient of each significant factor. The probability of axillary LN metastasis was calculated as follows: $1 /(1+\exp (-\mathrm{A}))$,

$\mathbf{a}$

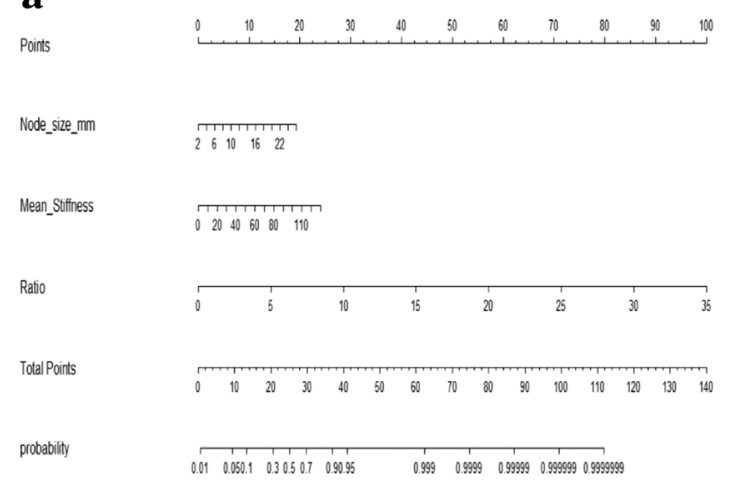

where $A=-4.9898+0.1498 \times($ nodal size $)+0.0345 \times($ mean stiffness $)+0.5321 \times($ ratio $)$

In the nomogram, the estimated probability of LN metastasis could be obtained by summing the scores of each variable and locating such on the total score scale. For instance, a patient with a $16-\mathrm{mm}$ nodal size (11 points), $70-\mathrm{kPa}$ mean stiffness (13 points), and 5 ratio (14 points) would score 38 points, which can be converted into $92.29 \%$ probability of $\mathrm{LN}$ metastasis (Fig. 2b).

The area under the curve (AUC) was 0.856 (95\% confidence interval (CI), 0.783-0.929) in the development cohort (Fig. 3a). The $p$ value obtained using the Hosmer-Lemeshow goodness-of-fit test was 0.354 , indicating a good fit of the model. When internal validation was performed using the 200 bootstrap samples, the mean absolute error was as low as 0.029 . Moreover, the calibration plots of the nomogram showed good agreement between the observed and predicted outcomes (Fig. 3b).

\section{Validation cohort}

In the validation cohort, 10 of the 80 patients had sentinel LN metastases, all of which were in the N1 stage. Tumor size of the patients with sentinel LN metastasis was larger than that of the patients without sentinel $\mathrm{LN}$ metastasis $(2.35 \mathrm{~cm}$ vs 1.61 $\mathrm{cm}, p=0.045)$. The other clinicopathologic factors were no significantly different between the patients with and without sentinel LN metastasis (Table 1). The median age of all patients was 55 (range, 22-76) years. The findings for estrogen receptor (ER) and human epidermal growth factor receptor 2 (HER2) were positive in $67(83.8 \%)$ and $15(18.8 \%)$ patients, respectively. T1 stage and grade 2 were the most common classifications in $52(65.0 \%)$ and $43(53.8 \%)$ patients, respectively. The number of elderly patients and patients with HER2-negative findings and low $\mathrm{N}$ stage was higher in the validation cohort than in the development cohort (Table 2).

\section{b}

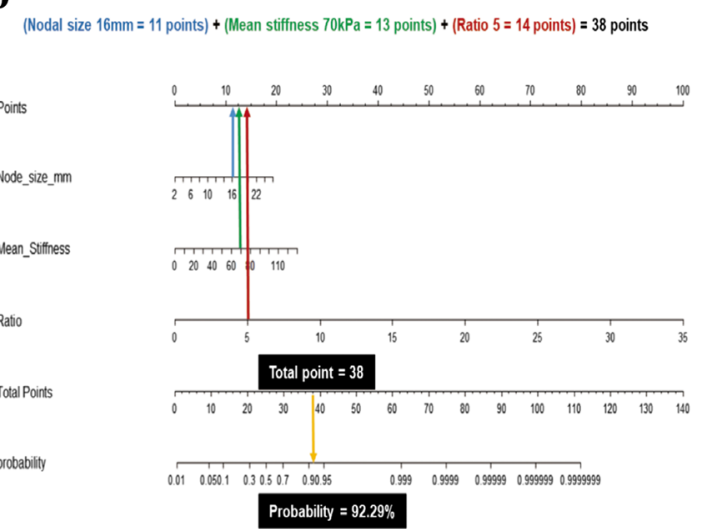

Fig. 2 Constructed nomogram. a Constructed nomogram based on the predictive factors of axillary LN metastasis, such as nodal size, mean stiffness, and elasticity ratio. b Example of constructed nomogram. LN, lymph node 
Fig. 3 ROC curve of the nomogram and the calibration plot. a ROC curve of the nomogram; $\mathbf{b}$ the calibration plot in the development cohort with internal validation; $\mathbf{c}$ ROC curve of the nomogram; $\mathbf{d}$ the calibration plot in the validation cohort with external validation. ROC, receiver operating characteristic

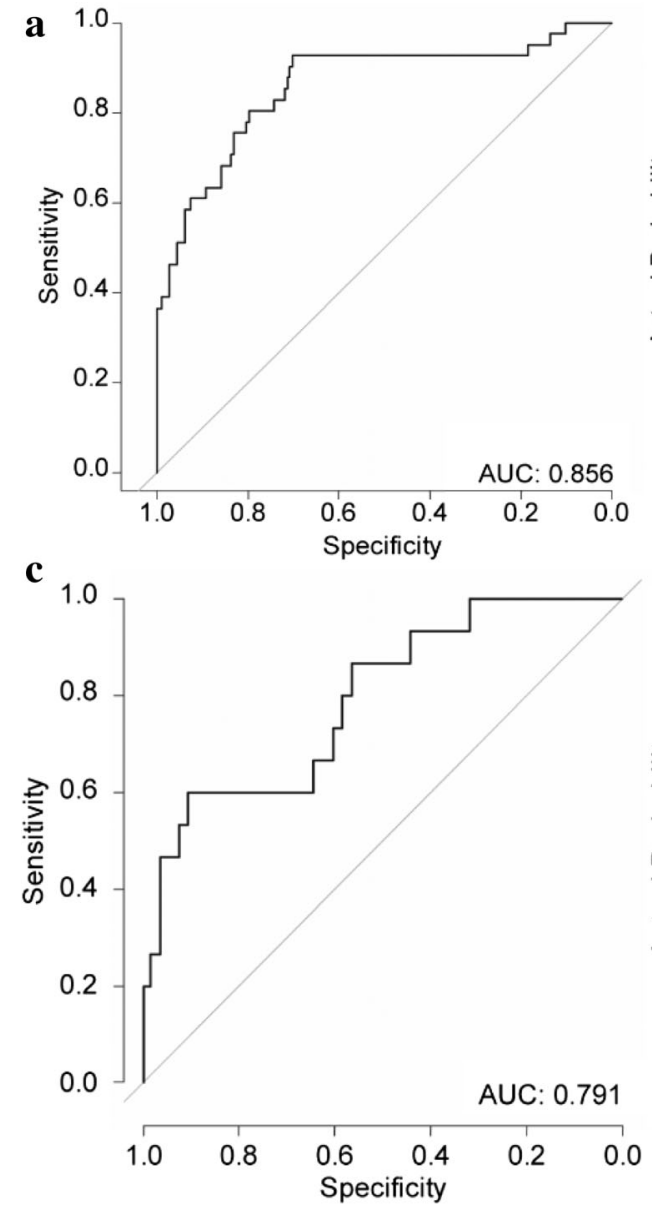

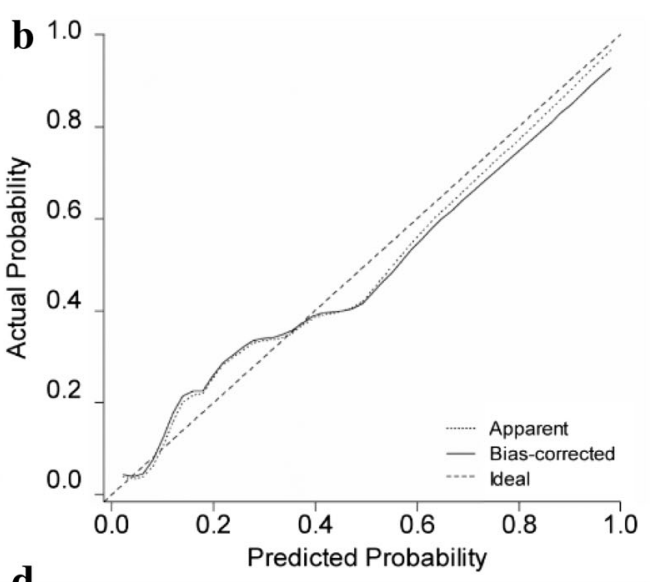

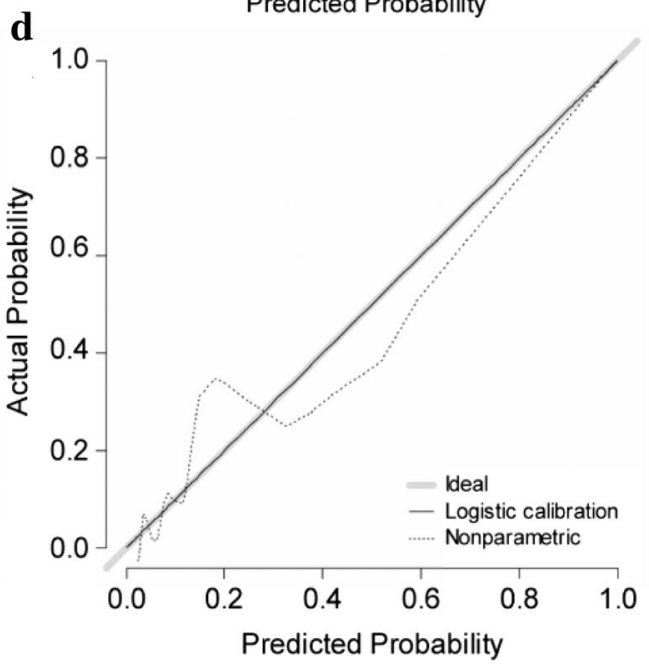

Of the 217 sentinel LNs, 15 (6.9\%) were metastatic, and $202(93.1 \%)$ were non-metastatic, including seven (3.2\%) micro-metastatic LNs. The metastatic LNs tended to be larger than non-metastatic LNs, but it was not significant $(8.70 \mathrm{~mm}$ vs $7.20 \mathrm{~mm}, p=0.123$ ) (Table 3 ). The mean stiffness of the metastatic sentinel LNs was significantly greater than that of the non-metastatic sentinel LNs (23.54 vs $10.41 \mathrm{kPa}, p=$ 0.005 ) (Fig. 4). Furthermore, the elasticity ratio of the metastatic sentinel LNs was significantly higher than that of the non-metastatic sentinel LNs (3.24 vs $1.49, p=0.028$ ). Meanwhile, there was no difference in nodal size and elasticity values according to histological types of breast cancer (Table 4).

\section{External validation of the nomogram}

The nomogram was validated with the independent set including only 217 sentinel LNs. The discriminative power was good, with an AUC of 0.791 (95\% CI, 0.668-0.915) (Fig. $3 \mathrm{c})$. The calibration plots of the nomogram showed good agreement between the observed and predicted outcomes (Fig. 3d).

\section{Discussion}

In this study, we generated a nomogram to predict metastatic LNs based on nodal size and elasticity values such as mean stiffness and elasticity ratio of harvested LNs on B-mode ultrasound with ex vivo SWE using data from our previous study. Thereafter, we tested the predictive ability of the nomogram in an independent set consisting of sentinel LNs. The performance of constructed nomogram to predict sentinel LNs metastasis was high in terms of good discrimination and calibration.

Cancer researchers, clinicians, and the public are becoming increasingly interested in statistical models designed to predict the occurrence or the outcome of cancer, along with the efficacy of treatments [25-27]. Among several prediction models, nomograms have been shown to provide personalized reasonable risk estimates that facilitate management-related decisions [27]. Indeed, our nomogram can be used to calculate the probability of each axillary LN metastasis easily and rapidly. When the total point calculated using the nodal size, mean stiffness, and elasticity ratio is over 35 points, the probability of LN metastasis exceeds $90 \%$. If the total point is over 48 points, the probability of LN metastasis exceeds $99 \%$. 
Table 1 Clinicopathologic characteristics of the validation cohort according to LN metastasis

\begin{tabular}{|c|c|c|c|c|}
\hline Variables & $\begin{array}{l}\text { Patients with non-malignant } \\
\text { sentinel LN }(N=70)\end{array}$ & $\begin{array}{l}\text { Patients with malignant } \\
\text { sentinel LN }(N=10)\end{array}$ & $\begin{array}{l}\text { All patients } \\
(N=80)\end{array}$ & $p$ value \\
\hline Median age (years) & $55.5(35-76)$ & $54(22-75)$ & $55(22-76)$ & 0.716 \\
\hline Tumor size $(\mathrm{cm})$ & $1.61 \pm 1.07$ & $2.35 \pm 1.14$ & $1.68 \pm 1.06$ & 0.045 \\
\hline T stage & & & & $0.520^{\dagger}$ \\
\hline $\mathrm{T} 1 \mathrm{mi}$ & $2(2.9 \%)$ & 0 & $2(2.5 \%)$ & \\
\hline $\mathrm{T} 1$ & $47(67.1 \%)$ & $5(50.0 \%)$ & $52(65.0 \%)$ & \\
\hline $\mathrm{T} 2$ & $20(28.6 \%)$ & $5(50.0 \%)$ & $25(31.3 \%)$ & \\
\hline $\mathrm{T} 3$ & $1(1.4 \%)$ & 0 & $1(1.2 \%)$ & \\
\hline HG & & & & $0.312^{\dagger}$ \\
\hline Grade I & $18(25.7 \%)$ & $1(10.0 \%)$ & $19(23.8 \%)$ & \\
\hline Grade II & $38(54.3 \%)$ & $5(50.0 \%)$ & $43(53.8 \%)$ & \\
\hline Grade III & $14(20.0 \%)$ & $4(40.0 \%)$ & $18(22.5 \%)$ & \\
\hline ER & & & & $0.662^{\dagger}$ \\
\hline Positive & $59(84.3 \%)$ & $8(80.0 \%)$ & $67(83.8 \%)$ & \\
\hline Negative & $11(15.7 \%)$ & $2(20.0 \%)$ & $13(16.2 \%)$ & \\
\hline PR & & & & $0.715^{\dagger}$ \\
\hline Positive & $48(68.6 \%)$ & $8(80.0 \%)$ & $56(70.0 \%)$ & \\
\hline Negative & $22(31.4 \%)$ & $2(20.0 \%)$ & $24(30.0 \%)$ & \\
\hline HER2 & & & & $0.086^{\dagger}$ \\
\hline Positive & $11(15.7 \%)$ & $4(40 \%)$ & $15(18.8 \%)$ & \\
\hline Negative & $59(84.3 \%)$ & $6(60 \%)$ & $65(81.3 \%)$ & \\
\hline
\end{tabular}

$L N$, lymph node; $H G$, histologic grade; $E R$, estrogen receptor; $P R$, progesterone receptor; $H E R 2$, human epidermal growth factor receptor 2

${ }^{\dagger}$ Fisher's exact test

\begin{tabular}{|c|c|c|c|}
\hline Characteristics & Development cohort $(N=55)$ & Validation cohort $(N=80)$ & $p$ value \\
\hline Median age, years (range) & $49(31-69)$ & $55(22-76)$ & $0.009^{*}$ \\
\hline Tumor size, cm (range) & $1.75(0.10-3.60)$ & $1.70(0.1-6.20)$ & 0.766 \\
\hline T stage & & & $0.382^{\dagger}$ \\
\hline Tis & $2(3.6 \%)$ & 0 & \\
\hline T1mi & $2(3.6 \%)$ & $2(2.5 \%)$ & \\
\hline $\mathrm{T} 1$ & $31(56.4 \%)$ & $52(65.0 \%)$ & \\
\hline $\mathrm{T} 2$ & $20(36.4 \%)$ & $25(31.3 \%)$ & \\
\hline $\mathrm{T} 3$ & 0 & $1(1.3 \%)$ & \\
\hline $\mathrm{N}$ stage & & & $<0.001^{\dagger}$ \\
\hline 0 & $28(51.0 \%)$ & $70(87.5 \%)$ & \\
\hline N1 & $19(34.5 \%)$ & $10(12.5 \%)$ & \\
\hline N2 & $6(11.0 \%)$ & 0 & \\
\hline N3 & $2(3.5 \%)$ & 0 & \\
\hline $\mathrm{HG}^{\mathrm{a}}$ & & & 0.096 \\
\hline Grade I & $7(12.7 \%)$ & $19(23.8 \%)$ & \\
\hline Grade II & $25(45.5 \%)$ & $43(53.8 \%)$ & \\
\hline Grade III & $20(36.4 \%)$ & $18(22.5 \%)$ & \\
\hline ER & & & 0.074 \\
\hline Positive & $39(70.9 \%)$ & $67(83.8 \%)$ & \\
\hline Negative & $16(29.1 \%)$ & $13(16.2 \%)$ & \\
\hline $\mathrm{PR}$ & & & 0.157 \\
\hline Positive & $32(58.2 \%)$ & $56(70.0 \%)$ & \\
\hline Negative & $23(41.8 \%)$ & $24(30.0 \%)$ & \\
\hline HER2 & & & 0.012 \\
\hline Positive & $21(38.2 \%)$ & $15(18.8 \%)$ & \\
\hline Negative & $34(61.8 \%)$ & $65(81.3 \%)$ & \\
\hline
\end{tabular}

$H G$, histologic grade; $E R$, estrogen receptor; $P R$, progesterone receptor; HER2, human epidermal growth factor receptor 2

*Mann-Whitney test

${ }^{\dagger}$ Fisher's exact test

${ }^{\mathrm{a}}$ Missing data 
Table 3 Ultrasound features and elasticity values measured using intraoperative ex vivo shear-wave elastography and total points in the nomogram between the nonmetastatic and metastatic axillary LNs

\begin{tabular}{lcclc}
\hline & $\begin{array}{l}\text { Total } \\
(n=217)\end{array}$ & $\begin{array}{l}\text { Non-metastatic } \\
\text { sentinel LNs } \\
(n=202)\end{array}$ & $\begin{array}{l}\text { Metastatic sentinel LNs } \\
(n=15)\end{array}$ & $p$ value \\
\hline Nodal size (mm) & $7.31 \pm 3.63$ & $7.20 \pm 3.62$ & $8.70 \pm 3.60$ & 0.123 \\
Mean stiffness (kPa) & $11.32 \pm 7.2$ & $10.41 \pm 5.24$ & $23.54 \pm 15.28$ & 0.005 \\
Elasticity ratio & $1.61 \pm 1.12$ & $1.49 \pm 0.77$ & $3.24 \pm 2.76$ & 0.028 \\
Total points in nomogram & $9.08(95 \% \mathrm{CI}$, & $\begin{array}{c}7.67(95 \% \mathrm{CI}, \\
6.69-8.64)\end{array}$ & $28.06(95 \% \mathrm{CI}, 11.29-44.83)$ & $<0.001^{*}$ \\
& $7.54-10.62)$ & & \\
\hline
\end{tabular}

$L N$, lymph node

*Mann-Whitney test
Indeed, the mean total point in the nomogram was significantly different between the non-metastatic and metastatic sentinel LNs (Supplementary Table 3). In the metastatic sentinel LNs, the probability of LN metastasis was approximately $70 \%$, as the mean total point was 28 points; in the non-metastatic sentinel LNs, the probability of LN metastasis was less than $10 \%$, as the mean total point was 8 points.

The discriminative power of the nomogram was quantified using the AUC exhibiting the accuracy of the test, with an AUC of 0.5 being defined as non-informative; $0.5-0.7$, fair; $0.7-0.9$, good; and $>0.9$, excellent. Our nomogram revealed good discriminative power with the AUC of 0.856 in the development cohort and 0.791 in the validation cohort. In addition, the calibration plot showed good agreement between the observed and predicted probabilities in both the development and validation cohorts. Therefore, our constructed nomogram was suitable in predicting the probability of metastasis among the harvested sentinel LNs.

In the validation cohort, the ultrasound and SWE characteristics of sentinel LNs were not different between histologic types of breast cancer in line with previous report [28]. The mean stiffness and elasticity ratio of the metastatic sentinel LNs were significantly higher than those of the non- metastatic LNs. These findings are similar to those of the development cohort in our previous study. The nodal size tended to be larger in the metastatic LNs than in the nonmetastatic LNs, although the trend was not significant. It was presumed that the proportion of the metastatic LNs was higher in the development cohort than in the validation cohort (Table 2). Further research with a larger number of axillary LNs is needed to investigate the accurate relationship between the nodal size and LN metastasis.

Recently, the American College of Surgeons Oncology Group Z0011 prospective, randomized clinical trial changed the standard approach to axillary surgery, showing that the omission of ALND was possible in early breast cancer patients who underwent breast conserving surgery and adjuvant systemic therapy even with 1-2 metastatic sentinel LNs [29, 30]. Several previous trials also reported consistent findings with those of the Z0011 trial [2, 31]. Since these trials presented that ALND could be omitted even in patients with positive sentinel LNs, the need for intraoperative frozen section analysis of sentinel LNs is questionable. In fact, not only ALND but also intraoperative frozen section analysis of sentinel LNs in patients who underwent breast conservative surgery was declined after the Z0011 trial was published [32, 33].
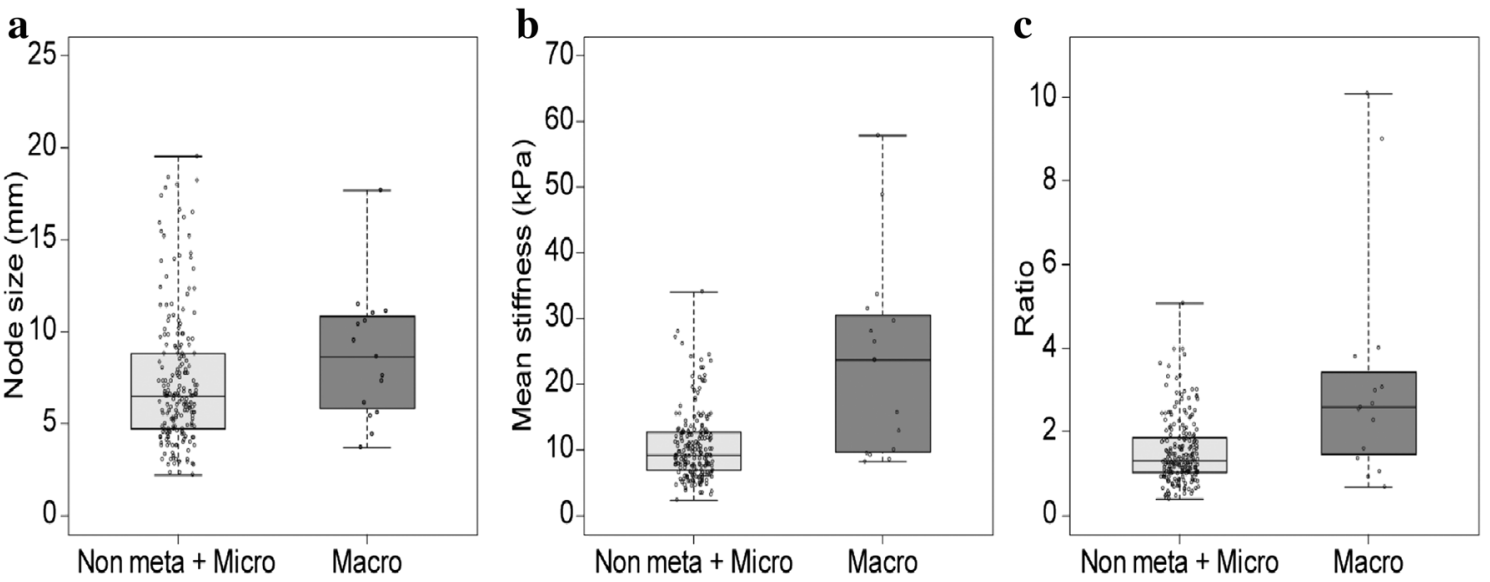

Fig. 4 Ultrasound feature and elasticity values between non-metastatic and metastatic lymph nodes. Box plot of the (a) nodal size, (b) mean stiffness, and (c) elasticity ratio between the non-metastatic and metastatic lymph nodes 
Table 4 Ultrasound features and elasticity values measured using intraoperative ex vivo shear-wave elastography according to histological types of breast cancer

\begin{tabular}{lcccc}
\hline & \multicolumn{2}{c}{ Histologic type } & & $p$ value \\
\cline { 2 - 4 } & IDC $(n=179)$ & $\operatorname{ILC}(n=12)$ & Others* $(n=26)$ & \\
\hline Nodal size $(\mathrm{mm})$ & $7.13 \pm 3.35$ & $7.43 \pm 4.99$ & $8.45 \pm 4.61$ & 0.225 \\
Mean stiffness $(\mathrm{kPa})$ & $11.53 \pm 7.56$ & $8.44 \pm 2.67$ & $11.15 \pm 5.82$ & 0.353 \\
Elasticity ratio & $1.60 \pm 1.18$ & $1.66 \pm 0.64$ & $1.66 \pm 0.75$ & 0.960 \\
\hline
\end{tabular}

$I D C$, invasive ductal carcinoma; $I L C$, invasive lobular carcinoma

*Others; medullary carcinoma $(n=3)$, mucinous carcinoma $(n=11)$, tubular carcinoma $(n=4)$, cribriform carcinoma $(n=5)$, apocrine carcinoma $(n=3)$
Furthermore, Noordaa et al suggested that omitting intraoperative pathologic assessment of LNs was a reasonable option in patients with a low nodal burden, such as clinically nodenegative breast cancer, who were treated with upfront surgery [34].

However, one of the major disadvantages of omitting intraoperative frozen section analysis is secondary surgery for ALND. To date, the exact rate of secondary surgery for ALND after skipping intraoperative frozen section analysis has not been well researched. Nevertheless, concerns of secondary operation for ALND can be reduced if intraoperative pathologic examination was performed for sentinel LNs in suspicion of metastasis, as identified by our nomogram.

Our study has some limitations. First, the cutoff value of nomogram to determine the intraoperative pathologic examination was not clarified in this analysis. Further prospective studies are needed to verify this issue. Second, the rate of metastatic LNs in the validation cohort was lower than that in the development cohort. It was probably because only sentinel LNs were included in the validation set. The number of metastatic LNs was 15 in the validation cohort of our study; nevertheless, these events were statistically sufficient to perform calibration and discrimination to warrant the use of our nomogram. Finally, concerns about the reproducibility of SWE still remained because interobserver or intraobserver agreements for the elasticity values were not assessed in this study. However, the other previous study showed reliable intraobserver and interobserver reproducibilities of SWE $[35,36]$. In addition, the reproducibility of SWE was expected to be higher in our study because specialized radiologists examined the LNs by ex vivo SWE that was less likely to interfere with surrounding tissue compared with in vivo SWE. Hence, it might be expected to precisely perform intraoperative ex vivo SWE of excised LNs within a short time.

In conclusion, our well-validated nomogram can be applied to predict nodal metastasis during SLNB. The clinical application of nomogram, which is mainly based on ex vivo SWE values, may help in reducing unnecessary intraoperative frozen section analysis as well as secondary operation rate in breast cancer patients who underwent SLNB.
Acknowledgements The authors appreciate the Biostatistics Collaboration Unit at Yonsei University College of Medicine for statistical analysis and consultation.

Funding information This work was supported by a grant from the research fund of Yonsei University Medical College, Seoul, Republic of Korea (6-2014-0199).

\section{Compliance with Ethical Standards}

Guarantor The scientific guarantor of this publication is Joon Jeong.

Conflict of Interest The authors of this manuscript declare no relationships with any companies, whose products or services may be related to the subject matter of the article.

Statistics and Biometry Biostatistics Collaboration Unit at Yonsei University College of Medicine has been consulted for statistical analysis.

Informed Consent Written informed consent was obtained from all patients in this study.

Ethical Approval Institutional Review Board approval was obtained.
Methodology
- prospective
- diagnostic study
- performed at one institution

Open Access This article is distributed under the terms of the Creative Commons Attribution 4.0 International License (http:// creativecommons.org/licenses/by/4.0/), which permits unrestricted use, distribution, and reproduction in any medium, provided you give appropriate credit to the original author(s) and the source, provide a link to the Creative Commons license, and indicate if changes were made.

\section{References}

1. McGuire WL (1987) Prognostic factors for recurrence and survival in human breast cancer. Breast Cancer Res Treat 10:5-9

2. Galimberti V, Cole BF, Zurrida S et al (2013) Axillary dissection versus no axillary dissection in patients with sentinel-node micrometastases (IBCSG 23-01): a phase 3 randomised controlled trial. Lancet Oncol 14:297-305

3. Lyman GH, Somerfield MR, Bosserman LD, Perkins CL, Weaver DL, Giuliano AE (2017) Sentinel lymph node biopsy for patients 
with early-stage breast cancer: American Society of Clinical Oncology Clinical Practice guideline update. J Clin Oncol 35: 561-564

4. Veronesi U, Viale G, Paganelli G et al (2010) Sentinel lymph node biopsy in breast cancer: ten-year results of a randomized controlled study. Ann Surg 251:595-600

5. Veronesi U, Paganelli G, Viale G et al (2003) A randomized comparison of sentinel-node biopsy with routine axillary dissection in breast cancer. N Engl J Med 349:546-553

6. Mansel RE, Fallowfield L, Kissin M et al (2006) Randomized multicenter trial of sentinel node biopsy versus standard axillary treatment in operable breast cancer: the ALMANAC Trial. J Natl Cancer Inst 98:599-609

7. Krag DN, Anderson SJ, Julian TB et al (2010) Sentinel-lymphnode resection compared with conventional axillary-lymph-node dissection in clinically node-negative patients with breast cancer: overall survival findings from the NSABP B-32 randomised phase 3 trial. Lancet Oncol 11:927-933

8. Youk JH, Son EJ, Gweon HM, Kim H, Park YJ, Kim JA (2014) Comparison of strain and shear wave elastography for the differentiation of benign from malignant breast lesions, combined with Bmode ultrasonography: qualitative and quantitative assessments. Ultrasound Med Biol 40:2336-2344

9. Lee SH, Chang JM, Cho N et al (2014) Practice guideline for the performance of breast ultrasound elastography. Ultrasonography 33:3-10

10. Goddi A, Bonardi M, Alessi S (2012) Breast elastography: a literature review. J Ultrasound 15:192-198

11. Kapetas P, Clauser P, Woitek R et al (2019) Quantitative multiparametric breast ultrasound: application of contrastenhanced ultrasound and elastography leads to an improved differentiation of benign and malignant lesions. Invest Radiol 54:257264

12. Lee SH, Chung J, Choi HY et al (2017) Evaluation of screening USdetected breast masses by combined use of elastography and color Doppler US with B-mode US in women with dense breasts: a multicenter prospective study. Radiology 285:660-669

13. Bae JS, Chang JM, Lee SH, Shin SU, Moon WK (2017) Prediction of invasive breast cancer using shear-wave elastography in patients with biopsy-confirmed ductal carcinoma in situ. Eur Radiol 27:715

14. Ma Y, Zhang S, Li J, Li J, Kang Y, Ren W (2017) Comparison of strain and shear-wave ultrasounic elastography in predicting the pathological response to neoadjuvant chemotherapy in breast cancers. Eur Radiol 27:2282-2291

15. Tourasse C, Denier JF, Awada A, Gratadour AC, Nessah-Bousquet K, Gay J (2012) Elastography in the assessment of sentinel lymph nodes prior to dissection. Eur J Radiol 81:3154-3159

16. Evans A, Rauchhaus P, Whelehan P et al (2014) Does shear wave ultrasound independently predict axillary lymph node metastasis in women with invasive breast cancer? Breast Cancer Res Treat 143: $153-157$

17. Kilic F, Velidedeoglu M, Ozturk T et al (2016) Ex vivo assessment of sentinel lymph nodes in breast cancer using shear wave elastography. J Ultrasound Med 35:271-277

18. Youk JH, Son EJ, Kim JA, Gweon HM (2017) Pre-operative evaluation of axillary lymph node status in patients with suspected breast cancer using shear wave elastography. Ultrasound Med Biol 43:1581-1586

19. Xu Y, Bai X, Chen Y et al (2018) Application of real-time elastography ultrasound in the diagnosis of axillary lymph node metastasis in breast cancer patients. Sci Rep 8:10234

20. Bae SJ, Park JT, Park AY et al (2018) Ex vivo shear-wave elastography of axillary lymph nodes to predict nodal metastasis in patients with primary breast cancer. J Breast Cancer 21:190-196
21. Barr RG, Zhang Z (2015) Shear-wave elastography of the breast: value of a quality measure and comparison with strain elastography. Radiology 275:45-53

22. Youk JH, Gweon HM, Son EJ (2017) Shear-wave elastography in breast ultrasonography: the state of the art. Ultrasonography 36 : 300-309

23. Dabbs DJ, Fung M, Landsittel D, McManus K, Johnson R (2004) Sentinel lymph node micrometastasis as a predictor of axillary tumor burden. Breast J 10:101-105

24. Iasonos A, Schrag D, Raj GV, Panageas KS (2008) How to build and interpret a nomogram for cancer prognosis. J Clin Oncol 26: 1364-1370

25. Rouzier R, Coutant $C$, Incitti R et al (2009) Breast cancer: nomograms to predict pathologic response after preoperative chemotherapy. J Med Liban 57:89-92

26. Graesslin O, Abdulkarim BS, Coutant C et al (2010) Nomogram to predict subsequent brain metastasis in patients with metastatic breast cancer. J Clin Oncol 28:2032-2037

27. Shariat SF, Capitanio U, Jeldres C, Karakiewicz PI (2009) Can nomograms be superior to other prediction tools? BJU Int 103: 492-495 discussion 495-497

28. Evans A, Sim YT, Thomson K, Jordan L, Purdie C, Vinnicombe SJ (2016) Shear wave elastography of breast cancer: sensitivity according to histological type in a large cohort. Breast 26:115-118

29. Giuliano AE, McCall L, Beitsch P et al (2010) Locoregional recurrence after sentinel lymph node dissection with or without axillary dissection in patients with sentinel lymph node metastases: the American College of Surgeons Oncology Group Z0011 randomized trial. Ann Surg 252:426-432 discussion 432-423

30. Giuliano AE, Ballman K, McCall L et al (2016) Locoregional recurrence after sentinel lymph node dissection with or without axillary dissection in patients with sentinel lymph node metastases: long-term follow-up from the American College of Surgeons Oncology Group (Alliance) ACOSOG Z0011 randomized trial. Ann Surg 264:413-420

31. Donker M, van Tienhoven G, Straver ME et al (2014) Radiotherapy or surgery of the axilla after a positive sentinel node in breast cancer (EORTC 10981-22023 AMAROS): a randomised, multicentre, open-label, phase 3 non-inferiority trial. Lancet Oncol 15:13031310

32. Bishop JA, Sun J, Ajkay N, Sanders MA (2016) Decline in frozen section diagnosis for axillary sentinel lymph nodes as a result of the American College of Surgeons Oncology Group Z0011 Trial. Arch Pathol Lab Med 140:830-835

33. Jorns JM, Kidwell KM (2016) Sentinel lymph node frozen-section utilization declines after publication of American College of Surgeons Oncology Group Z0011 trial results with no change in subsequent surgery for axillary lymph node dissection. Am J Clin Pathol 146:57-66

34. van der Noordaa MEM, Vrancken Peeters M, Rutgers EJT (2017) The intraoperative assessment of sentinel nodes - standards and controversies. Breast 34(Suppl 1):S64-S69

35. Cosgrove DO, Berg WA, Dore CJ et al (2012) Shear wave elastography for breast masses is highly reproducible. Eur Radiol 22:1023-1032

36. Kishimoto R, Kikuchi K, Koyama A et al (2019) Intra- and interoperator reproducibility of US point shear-wave elastography in various organs: evaluation in phantoms and healthy volunteers. Eur Radiol. https://doi.org/10.1007/s00330-019-06195-8

Publisher's note Springer Nature remains neutral with regard to jurisdictional claims in published maps and institutional affiliations. 